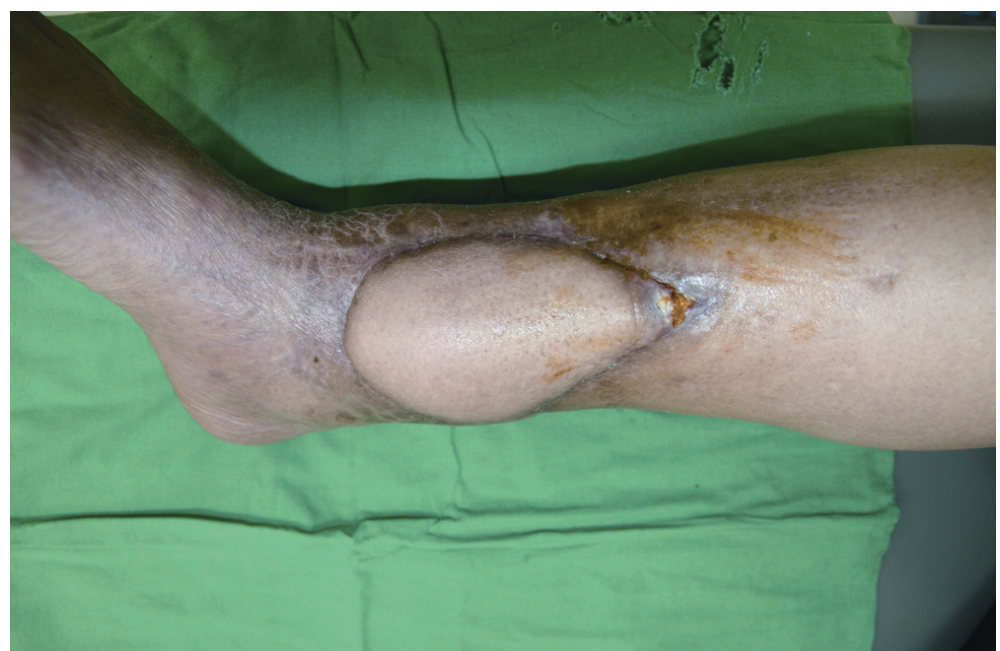

Fig. 4 .

We reconstructed the defect with an anterolateral thigh flap using the posterior tibial artery as the recipient vessel.

\section{Surgical Removal of Intraneural Perineurioma Arising in the Brachial Plexus Using an Interfascicular Dissection Technique}

Jae-Hyun Chung, Seong-Ho Jeong, Eun-Sang Dhong, Seung-Kyu Han

Department of Plastic Surgery, Korea University Guro Hospital, Korea University College of Medicine, Seoul, Korea

Correspondence: Seong-Ho Jeong

Department of Plastic Surgery, Korea University Guro Hospital, Korea University College of Medicine, 148 Gurodong-ro, Guro-gu, Seoul 152-703, Korea

Tel: +82-2-2626-3133, Fax: +82-2-852-7484

Email: surgilearn@korea.ac.kr

This article was presented as a poster at The 3rd Research and Reconstructive Forum on May 9-10, 2013 in Daegu, Korea

No potential conflict of interest relevant to this article was reported.

Received: 21 Jul 2013 • Revised: 11 Sep 2013 • Accepted: 27 Sep 2013 pISSN: 2234-6163・ elSSN: 2234-6171

http://dx.doi.org/10.5999/aps.2014.41.3.296• Arch Plast Surg 2014;41:296-299

Copyright (C) 2014 The Korean Society of Plastic and Reconstructive Surgeons

This is an Open Access article distributed under the terms of the Creative Commons

Attribution Non-Commercial License (http://creativecommons.org/licenses/by-nc/3.0)

which permits unrestricted non-commercial use, distribution, and reproduction in any medium, provided the original work is properly cited.

secondary operation was performed 18 months after successful reconstruction using a latissimus dorsi muscle flap. There is controversy concerning flap neovascularization, and many factors can compromise flap vascularity including the flap itself, patient comorbidities, and the wound condition. Given these risks, additional procedures affecting the flap should be performed with considerable care, with attention paid to flap vascularity.

\section{References}

1. Young CM. The revascularization of pedicle skin flaps in pigs: a functional and morphologic study. Plast Reconstr Surg 1982;70:455-64.

2. Klingenstrom P, Nylen B. Timing of transfer of tubed pedicles and cross-flaps. Plast Reconstr Surg 1966;37:1-

12.

3. Bhat W, Wiper JD, Platt AJ. Delayed autologous free anterolateral thigh flap failure. J Plast Reconstr Aesthet Surg 2011;64:420-1.

4. Kumar K, Jaffe W, London NJ, et al. Free flap neovascularization: myth or reality? J Reconstr Microsurg 2004;20:31-4.

5. Salgado CJ, Smith A, Kim S, et al. Effects of late loss of arterial inflow on free flap survival. J Reconstr Microsurg 2002;18:579-84.
A perineurioma is an uncommon neoplasm defined as a peripheral nerve sheath tumor composed exclusively of neoplastic perineurial cells, and its two main forms are intraneural and extraneural types [1]. Intraneural perineuriomas often cause motor or sensory deficits [2] and seem to be less common than extraneural ones. As intraneural perineurioma is rarely known to arise in the brachial plexus, principles of its surgical treatment have not been formulated thus far. Here, we report a rare case of surgical removal of intraneural perineurioma arising in the brachial plexus using a microsurgical interfascicular dissection technique for functional preservation. The technical feasibility and advantages of this method are discussed in this report.

A 58-year-old woman was referred for the surgical management of a right axillary mass that was incidentally noted on ultrasonography. The tumor had already been diagnosed as a spindle cell tumor from an ultrasound-guided biopsy. Spindle cell tumors are soft tissue sarcomas, which require a histopathological examination because of the limited material availability in the needle aspirates [3]. To rule out malignant 
peripheral nerve sheath tumors, we decided on the microsurgical removal of the tumor.

Under general anesthesia, the brachial plexus was approached via an infraclavicular approach. After ligation of the cephalic vein on the deltopectoral groove, the pectoralis minor muscle was divided. Below the pectoralis minor, the clavipectoral fascia was incised and the cords of the brachial plexus were inspected. The tumor originated from the sheath of the axillary nerve, which is one of the branches of the posterior cord and was found to be involved with the fascicles of the axillary nerve. It was difficult to differentiate pathologic fascicles from normal fascicles with the unaided eye (Fig. 1). Interfascicular dissection was performed with microsurgical techniques under high magnification. First, we exposed the healthy proximal portion of the axillary nerve and then differentiated each fascicle via internal neurolysis. Interfascicular dissection progressed distally toward the proximal edge of the tumor. The fascicle invaded by the tumor was very soft, brittle, and pulpy; further, it was dusky brown in color. In addition, these fascicles responded poorly to electrical stimulation by a nerve stimulator, while stimulation to the preserved fascicles caused contraction of the deltoid muscle. On the basis of these findings, we differentiated pathologic lesions from the healthy fascicles and removed the tumor with the preservation of the healthy portion of the nerve fascicles (Fig. 2).

The mass was $0.5 \mathrm{~cm} \times 1.0 \mathrm{~cm} \times 4.5 \mathrm{~cm}$ in size and was well circumscribed but unencapsulated (Fig. 3).
Upon histological examination, irregularly enlarged, hypercellular nerve fascicles containing spindled perineurial cells were seen arranged in pseudo-onion bulb whorls on the cross section. Upon immunohistochemical staining, endothelial membrane antigen-positive perineurial cells were seen arranged in pseudo-onion bulb whorls (Fig. 4) around S100-positive Schwann cells (Fig. 5). The tumor was eventually diagnosed as an intraneural perineurioma.

Intraneural perineuriomas most commonly involve the sciatic nerve or its branches, but the tumor can occur in the brachial plexus, trigeminal nerves, and the lumbosacral plexus [4]. Intraneural perineuriomas occur relatively rarely in the brachial plexus. Although a growing intraneural perineurioma can cause destruction of nerve fascicles and progressive loss of motor function, there has been no optimal management for this tumor because of its rare occurrence and insufficient clinical research.

In our case, it was very difficult to decide whether or not to remove the tumor because the patient had no subjective symptoms. However, we decided to remove the lesion and preserve the intact fascicles as much as possible in order to minimize neurologic complications. This was because the tumor could grow and give rise to functional deficits and a histologic examination was required to rule out malignancy. Nerve sheath tumors are usually removed en bloc except large tumors that necessitate excessive retraction of the surrounding fascicles to achieve an

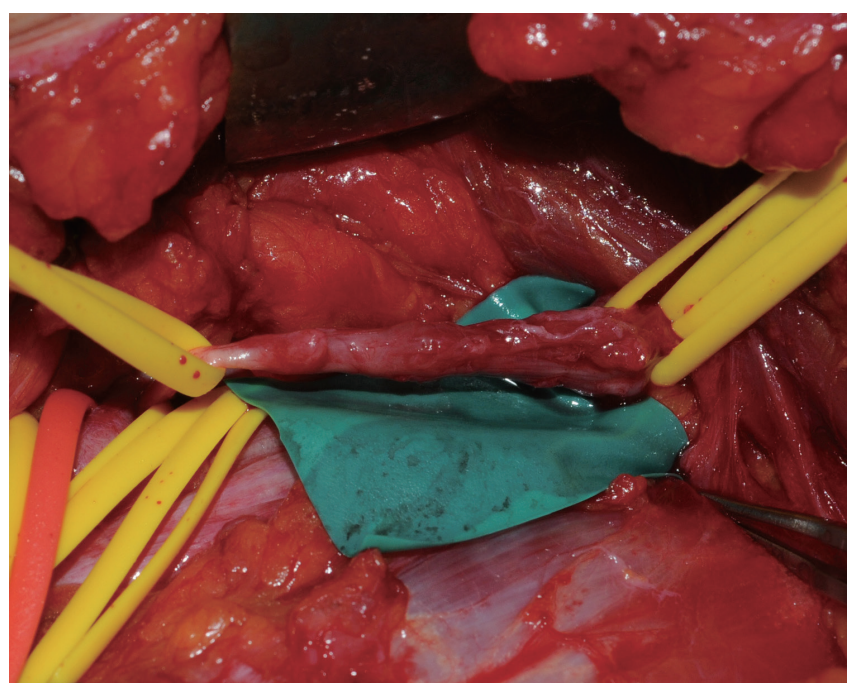

Fig. 1.

Intraoperative findings. The tumor originated from the axillary nerve of the posterior cord.

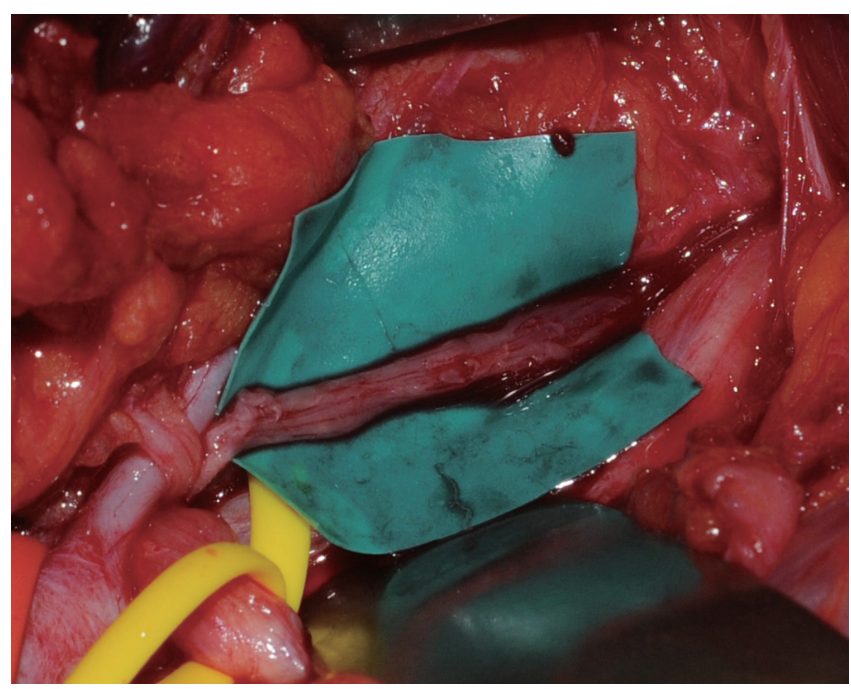

Fig. 2.

After tumor excision, one of the fascicle groups was preserved. 
en bloc resection [5]. Intraneural perineurioma in this case could be differentiated from the intact nerve fascicles via microscopic inspection and electrical stimulation and was systematically dissected from intact fascicles along a distinct plane between the normal and the pathologic fascicles. Consequently, we could achieve en bloc removal of the tumor. There is only one report of excising schwannoma or neurofibroma of the peripheral nerve by interfascicular dissection [5], and this is the first report in which intraneural perineurioma was resected selectively by this method.

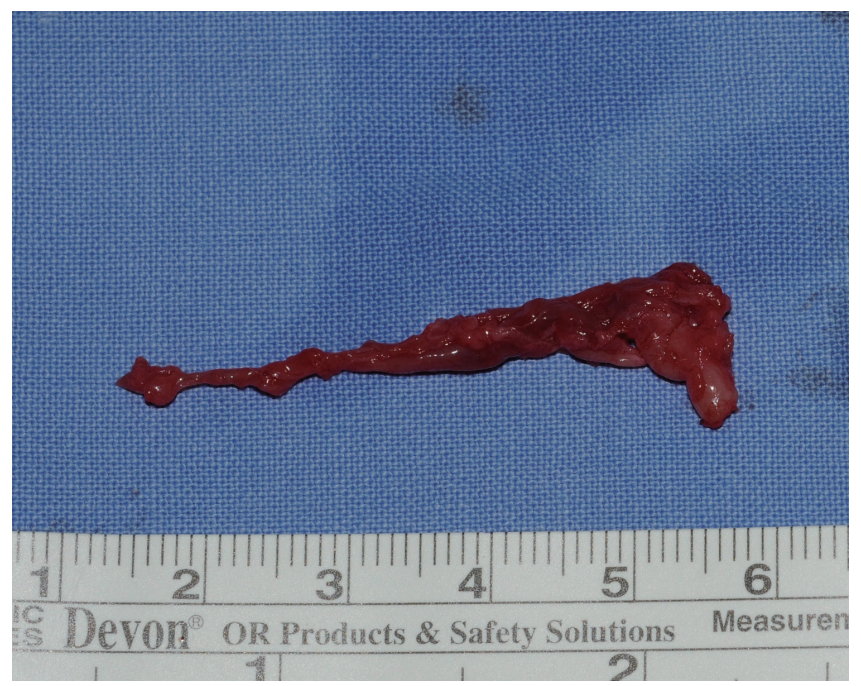

Fig. 3. The resected specimen measured about $0.5 \mathrm{~cm} \times 1.0 \mathrm{~cm} \times 4.5 \mathrm{~cm}$
Postoperatively, the patient suffered from temporary sensory and motor deficits, which were identified as axillary neuropathy by electromyography (EMG). It was expected that axillary neuropathy occurred because the intraoperative findings suggested that the tumor was involved with the fascicles of the axillary nerve. However, we assured the patient that she would recover fully, because the intact fascicles were confirmed intraoperatively. As expected, follow-up EMG at 11 months showed marked improvement. The axillary nerve divides into anterior and posterior branches. The anterior branch supplies the anterior part of the deltoid muscle and gives off a few small cutaneous branches. The posterior branch supplies the posterior part of the deltoid muscle and the teres minor muscle along with the skin over the lower two-thirds of the posterior part of this muscle, as well as that covering the long head of the triceps brachii. In our case, the initial needle EMG study showed abnormal spontaneous activity, no motor unit action potentials in the right deltoid muscle, and slightly reduced recruitment in the right teres minor muscle. The follow-up EMG at 6 months revealed complex polyphasic motor unit action potentials with discrete recruitment in the right deltoid muscle. This indicated that the axillary neuropathy was mainly limited to the anterior branch and that the degree of injury was very mild. The EMG result coincided with the operative procedure that the intact fascicles were preserved after tumor excision. Clinically, the patient gradually recovered during the

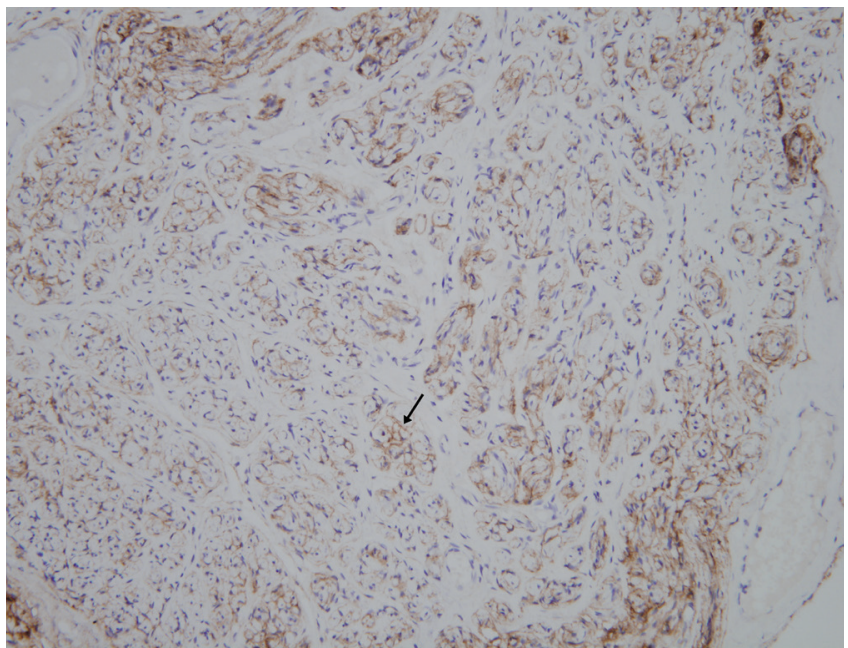

Fig. 4.

Upon immunohistochemical staining, the perineurial cells (black arrow) were positive for endothelial membrane antigen (endothelial membrane antigen, $\times 200)$.

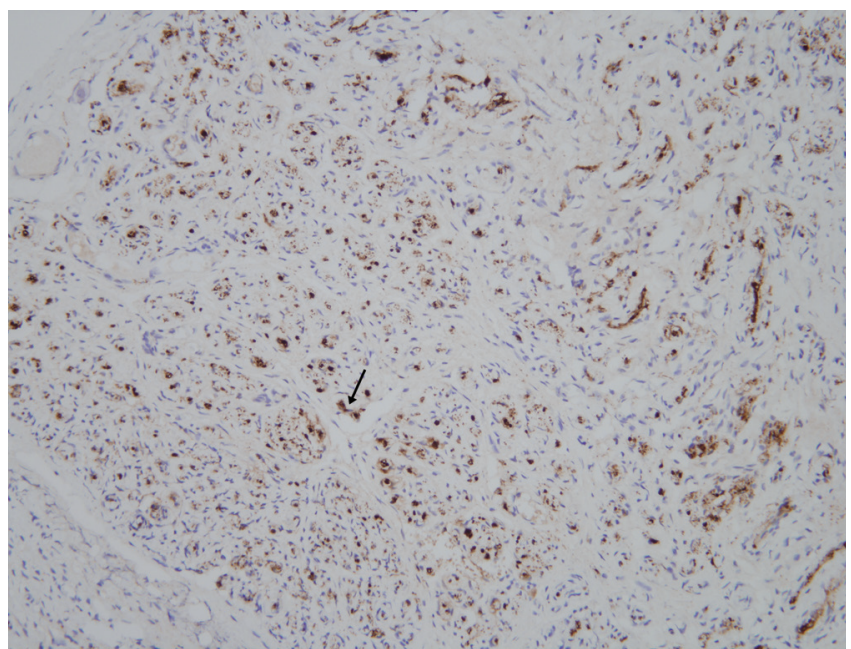

Fig. 5 .

Upon immunohistochemical staining, Schwann cells (black arrow) positive for $\mathrm{S} 100$ were surrounded by the perineurial cells $(\mathrm{S} 100, \times 200)$. 
18-month follow-up period and finally regained nearly normal shoulder function. The clinical course of this patient shows that the microsurgical removal of intraneural perineurioma in the brachial plexus is possible and can produce satisfactory results. We believe that this technique can be an effective and beneficial treatment for intraneural perineurioma arising from the brachial plexus.

\section{References}

1. Macarenco RS, Ellinger F, Oliveira AM. Perineurioma: a distinctive and underrecognized peripheral nerve sheath neoplasm. Arch Pathol Lab Med 2007;131:625-36.

2. Hornick JL, Fletcher CD. Intestinal perineuriomas: clinicopathologic definition of a new anatomic subset in a series of 10 cases. Am J Surg Pathol 2005;29:859-65.

3. Gupta K, Dey P, Vashisht R. Fine-needle aspiration cytology of malignant peripheral nerve sheath tumors. Diagn Cytopathol 2004;31:1-4.

4. Huang JH, Zaghloul K, Zager EL. Surgical management of brachial plexus region tumors. Surg Neurol 2004;61:372-8.

5. Russell SM. Preserve the nerve: microsurgical resection of peripheral nerve sheath tumors. Neurosurgery 2007;61:113-7.

\section{Diagnosis and Management of Ecthyma Gangrenosum in Chronic Renal Failure Patient}

Jordan D Frey ${ }^{1}$, Jo-Ann M Latkowski², Eddie Louie ${ }^{3}$, Ernest S Chiu ${ }^{1}$

${ }^{1}$ Institute of Reconstructive Plastic Surgery, ${ }^{2}$ Department of Dermatology, ${ }^{3}$ Department of Infectious Diseases, New York University Langone Medical Center, New York, NY, USA

Correspondence: Ernest S Chiu

Institute of Reconstructive Plastic Surgery, New York University Langone Medical, 240 East 38th Street, 13th Floor, New York, NY 10016, USA

Tel: +1-212-598-6500, Fax: +1-212-598-6889

E-mail: Ernest.Chiu@nyumc.org

This article contains supplemental Table S1.

No potential conflict of interest relevant to this article was reported.

Received: 28 Jan 2014 • Revised: 4 Mar 2014 • Accepted: 4 Mar 2014 pISSN: 2234-6163 • elSSN: 2234-6171

http://dx.doi.org/10.5999/aps.2014.41.3.299 • Arch Plast Surg 2014;41:299-301

Copyright (C) 2014 The Korean Society of Plastic and Reconstructive Surgeons

This is an Open Access article distributed under the terms of the Creative Commons

Attribution Non-Commercial License (http://creativecommons.org/licenses/by-nc/3.0/) which permits unrestricted non-commercial use, distribution, and reproduction in any medium, provided the original work is properly cited.
Ecthyma gangrenosum (EG) is an aggressive cutaneous disease caused by local or systemic Pseudomonas aeruginosa infection characterized by cutaneous manifestations ranging from maculopapular lesions and hemorrhagic bullae to necrotic tissue ulceration with surrounding erythema [1-5]. The differential diagnosis includes other causes of necrotic wounds such as calciphylaxis, septic emboli, cutaneous anthrax, cutaneous aspergillosis, and pyoderma gangrenosum. Individuals with EG are most often actively immunocompromised [3-5]. A high degree of clinical suspicion is required, but a tissue sample is ultimately necessary for definitive diagnosis. Current treatment recommendations include wide local excision as soon as a diagnosis is made concurrent with antibiotic therapy $[3,4]$. Reconstructive options vary and are largely dependent on the resultant defect's size, depth, and location [3]. While this disease has been described in various populations, the surgeon's role in EG is not well elucidated (Supplemental Table S1), notably in the adult population [1-5]. We present a case report emphasizing the surgeon's role in the diagnosis and management of EG in an adult with chronic renal failure.

A 46-year-old black woman with end-stage renal disease secondary to insulin dependent diabetes on hemodialysis for seven years, hypertension, peripheral vascular disease necessitating an above-knee amputation, and previous stroke presented to the Emergency Department with a 3 centimeter painless papular lesion on her midline upper back for

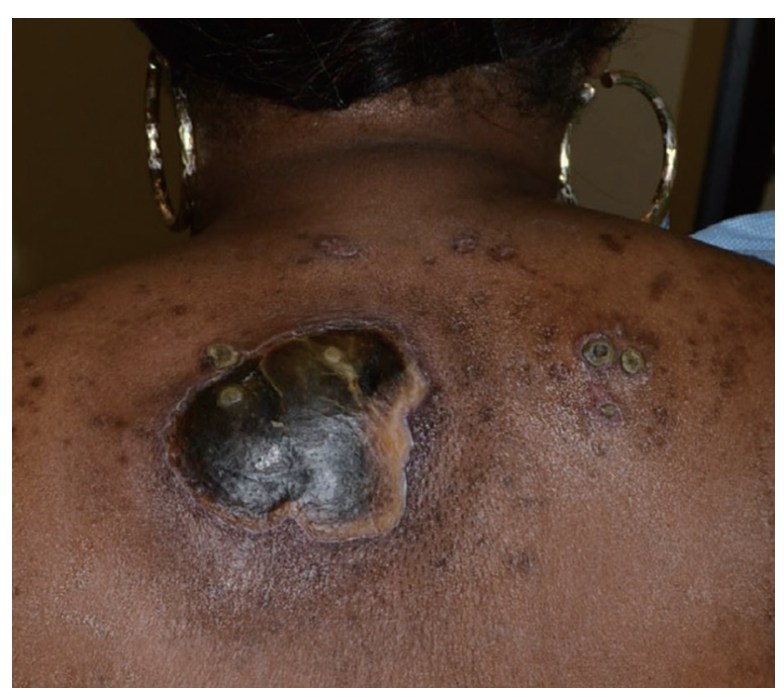

Fig. 1.

A 46-year-old black woman presenting with back lesion of 3 month's duration. 